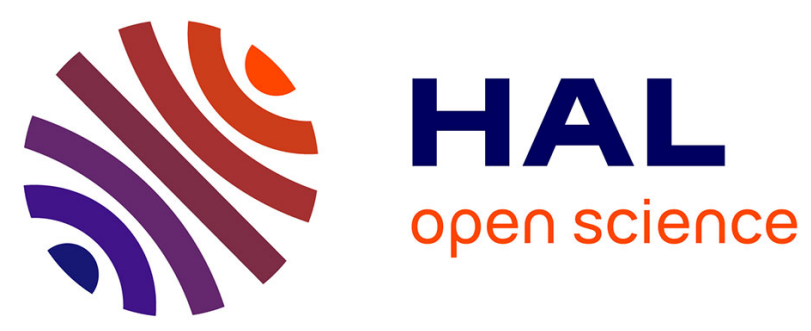

\title{
Touch-less Heartbeat Detection and Measurement-based Cardiopulmonary Modeling
}

\author{
Dany Obeid, Sawsan Sadek, Gheorghe I. Zaharia, Ghais El Zein
}

\section{To cite this version:}

Dany Obeid, Sawsan Sadek, Gheorghe I. Zaharia, Ghais El Zein. Touch-less Heartbeat Detection and Measurement-based Cardiopulmonary Modeling. Engineering in Medicine and Biology Society, Aug 2010, Buenos AIres, Argentina. pp.658-661, 10.1109/IEMBS.2010.5627214 . hal-00560211

\section{HAL Id: hal-00560211 https://hal.science/hal-00560211}

Submitted on 27 Jan 2011

HAL is a multi-disciplinary open access archive for the deposit and dissemination of scientific research documents, whether they are published or not. The documents may come from teaching and research institutions in France or abroad, or from public or private research centers.
L'archive ouverte pluridisciplinaire HAL, est destinée au dépôt et à la diffusion de documents scientifiques de niveau recherche, publiés ou non, émanant des établissements d'enseignement et de recherche français ou étrangers, des laboratoires publics ou privés. 


\title{
Touch-less Heartbeat Detection and Measurement-based Cardiopulmonary Modeling
}

\author{
D. Obeid ${ }^{1}$, S. Sadek ${ }^{2}$, G. Zaharia ${ }^{1}$, and G. El Zein ${ }^{1}$ \\ ${ }^{1}$ IETR UMR CNRS 6164 - INSA, Rennes, France, ${ }^{2}$ Lebanese University, IUT, Saida, Lebanon \\ dany.obeid@insa-rennes.fr
}

\begin{abstract}
This paper presents a system for touch-less heartbeat detection and a cardiopulmonary signal modeling approach. Using a vector network analyzer, a microwave system is tested for the detection of the heartbeat signal at a distance of $1 \mathrm{~m}$ from a person. The proposed system shows the ability of detecting the heartbeat signals with the possibility of tuning both frequency and power. Measurements are performed at 2.4, 5.8, 10, 16, and $60 \mathrm{GHz}$, as well as at different power levels between 0 and $-27 \mathrm{dBm}$. Based on measurements performed for both respiration and heart beatings, a model representing the cardiopulmonary activity is presented. The heartbeat rate and the heart rate variability are extracted from the modeling signal using wavelet and classic filters, for SNR between 0 and $-20 \mathrm{~dB}$.
\end{abstract}

\section{INTRODUCTION}

$\mathrm{T}$ HE utility of microwave Doppler radar used in home monitoring has recently increased. Electrocardiograms are perturbing for patients with conditions such as burn victims or newly born infants. Based on the Doppler theory, a target with a quasi-periodic movement reflects the transmitted signal with its phase modulated by the timevarying position of the target [1]. Hence, the reflected signal off the person's chest contains information about the chest displacement, due to heartbeat and respiration. On the other hand, while holding breath, the reflected signal depends on the chest displacement due to heartbeat alone. The chest displacement variation, caused by respiration, is between 4 and $12 \mathrm{~mm}$ [2]. However, the chest displacement due to heartbeat alone ranges between 0.2 and $0.5 \mathrm{~mm}$ [3]. Also at rest, the respiration rate corresponds to a frequency that varies between 0.1 and $0.3 \mathrm{~Hz}$, while the heartbeat rate corresponds to a frequency that varies within the $1-3 \mathrm{~Hz}$ interval [4].

Related work was intended to detect life signs $[5,6]$, respiration rates and heartbeat rates, using fixed frequency and fixed power of the transmitted signal. Operating at 1.6 $\mathrm{GHz}$ and $2.4 \mathrm{GHz}$, direct-conversion Doppler radars have been integrated in $0.25 \mu \mathrm{m}$ CMOS and BiCMOS technologies [7]. Heart and respiration activities were detected using a modified Wireless Local Area Network PCMCIA card, and a module combining the transmitted and reflected signals [8]. Other systems operating in the Ka-Band

The authors acknowledge CEDRE (project reference number: 08SciF6/L4), AUF (project reference number: G3-110/1649), and "Région Bretagne" for partially funding this work. were described in $[9,10]$ using a low power double-sideband transmission signal. Recently, a new study shows the possibility of detecting the presence of a preson through a wall using Ultra-Wideband (UWB) radar [11]. Some experiments are preformed to detect life signs using the 4-7 GHz band with $1 \mathrm{~mW}$ power and around $7 \mathrm{~dB}$ antenna gain [12]. Another system operating at $10 \mathrm{GHz}$ showed the ability to detect the heart and the respiration activity of a person behind a wall [13].

In this work, a new microwave system for heartbeat detection is proposed. In addition to the installation simplicity, the proposed system has the ability of tuning both frequency and power. This is useful in determining the optimal frequency with the minimum transmitted power before the implementation process. Note that the transmission of a signal with minimum power would be safer for both the patient and the medical staff. Based on real experiments, a model representing the cardiopulmonary activity is proposed. The separation of the heartbeat and the respiration signals is achieved using wavelet and classic filtering, and at low Signal to Noise Ratio (SNR). The rest of the paper is organized as follows: Sections II presents the proposed system and shows the heartbeat signals detected at different operational frequencies and different radiated powers. Section III states the proposed model representing the cardiopulmonary signal. Section IV shows the results in extracting the heartbeat rate and the Heart Rate Variability (HRV) upon applying several processing techniques to the modeling signals. Section V concludes the work.

\section{Multi-TunABle System}

\section{A. Proposed System}

The proposed system is based on using a Vector Network Analyzer (VNA) (Agilent N5230A 4-Port) operating up to $20 \mathrm{GHz}$, and two horn antennas. Many features are accessible by using a VNA, such as the choice of the sweep time and the number of measurement points; therefore, the sampling rate. In addition, the frequency and the radiated power of the transmitted signal can be set and modified, and the time variation of the phase of the transmission coefficient $\mathrm{S}_{21}$ can be measured. Due to the limited frequency $(20 \mathrm{GHz})$ of the VNA, an up-conversion method is used in order to obtain a $60 \mathrm{GHz}$ signal. The proposed system operating at 
several frequencies and the RF blocks of the $60 \mathrm{GHz}$ signal are described in details in [14].

The VNA generates a Continuous Wave (CW) signal at the desired frequency. The reflected signal off the person's chest is received by the antenna and fed back into the VNA, where the phase of $S_{21}$ is computed. This phase corresponds to the difference between the phase of the received and the transmitted signal. Measurements are performed at several frequencies: $2.4 \mathrm{GHz}, 5.8 \mathrm{GHz}, 10 \mathrm{GHz}, 16 \mathrm{GHz}$, and 60 GHz.

\section{B. Heartbeat Signals Detected at Different Frequencies}

At a distance of $1 \mathrm{~m}$ from a 27 years old person, and at -10 $\mathrm{dBm}$ total output power, the system was tested at different frequencies: $2.4 \mathrm{GHz}, 5.8 \mathrm{GHz}, 10 \mathrm{GHz}, 16 \mathrm{GHz}$, and 60 $\mathrm{GHz}$. The heartbeat signal detected at $2.4 \mathrm{GHz}$ is shown in Fig. 1(a) where a phase variation of $1.57^{\circ}$ is observed. The heartbeat signal detected at $5.8 \mathrm{GHz}$ is shown in Fig. 1(b) where a phase variation of $3.66^{\circ}$ is observed. Operating at 10 $\mathrm{GHz}$, our proposed system indicates a phase variation of $5.27^{\circ}$, as shown in Fig. 1(c). Operating at $16 \mathrm{GHz}$, our proposed system indicates a phase variation of $10.46^{\circ}$ as shown in Fig. 1(d). The heartbeat signal detected using 60 $\mathrm{GHz}$ signal is presented in Fig. 1(e) where a phase variation of $43.85^{\circ}$ is observed. The difference in the phase variation due to heartbeat, detected at different frequencies, is shown in Fig. 1(f) where the heartbeat signals for different frequencies are plotted with the same scale. From top to bottom, Fig. 1(f) shows the heartbeat signal detected from $2.4 \mathrm{GHz}$ to $60 \mathrm{GHz}$. Higher sensitivity to small displacement is obtained at higher frequencies.

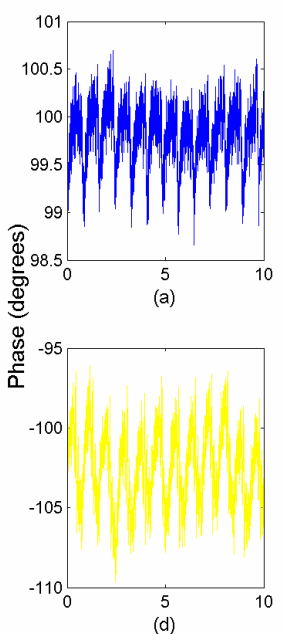

(d)
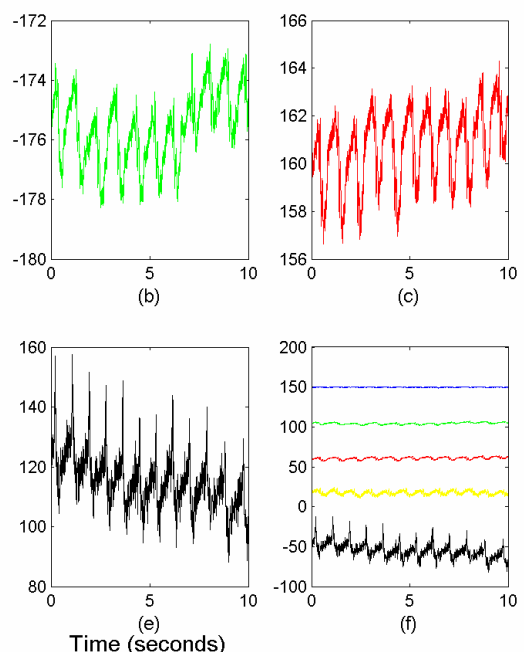

Fig. 1. Phase variations due to heartbeats detected at $2.4 \mathrm{GHz}$ (a), 5.8 $\mathrm{GHz}$ (b), $10 \mathrm{GHz}$ (c), $16 \mathrm{GHz}$ (d), and $60 \mathrm{GHz}$ (e).

\section{Heartbeat Signals Detected at Different Radiated Powers}

In order to avoid any possible interaction between the electromagnetic radiation and the human organism, and after verifying the feasibility of the proposed system, the next step was to minimize the radiated power. This includes both the transmitted power and the antenna gain. Operating at 2.4 $\mathrm{GHz}$, the proposed system shows the capability to detect body motions due to heart-beating at a radiated power as low as $-27 \mathrm{dBm}$; this value corresponds to the generated power of the VNA $(-40 \mathrm{dBm})$ added to the antenna gain $(\sim 13 \mathrm{~dB})$. Starting at $-2 \mathrm{dBm}$ and decreasing the power by a step of 5 $\mathrm{dB}$ down to $-27 \mathrm{dBm}$, our system still detects the heart activity, as shown in Fig. 2. Fig. 2(a-f) show respectively, the heartbeat signal detected using $2.4 \mathrm{GHz}$ operational frequency and for a radiated power of $-2 \mathrm{dBm},-7 \mathrm{dBm},-12$ $\mathrm{dBm},-17 \mathrm{dBm},-22 \mathrm{dBm}$, and $-27 \mathrm{dBm}$.
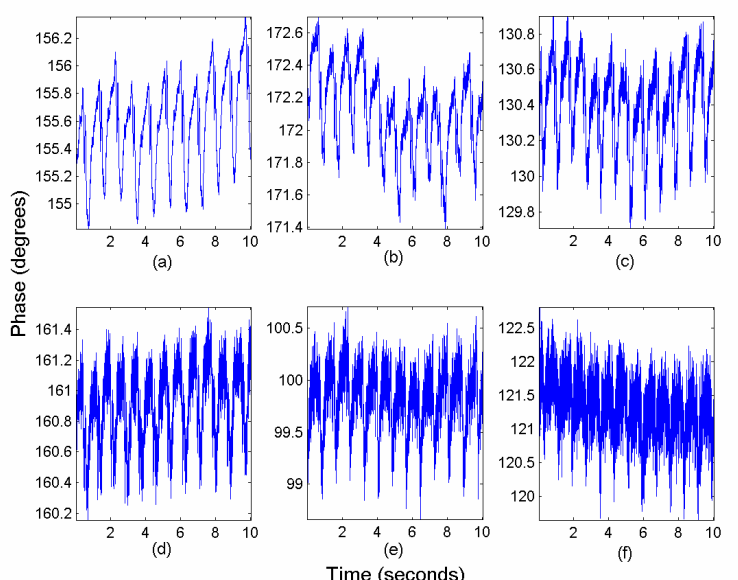

Fig. 2. Phase variations due to heartbeats detected at $2.4 \mathrm{GHz}$ with radiated power of $-2 \mathrm{dBm}$ (a), $-7 \mathrm{dBm}$ (b), $-12 \mathrm{dBm}$ (c), $-17 \mathrm{dBm}(\mathrm{d})$, $-22 \mathrm{dBm}(\mathrm{e})$, and $-27 \mathrm{dBm}(\mathrm{f})$

Even though the heartbeat signal detected at $-27 \mathrm{dBm}$ shows low SNR, the peaks of the signal are detectable. It is noteworthy to mention that the presented results are prior to any filtering or smoothing techniques.

\section{CARDIOPULMONARY MODELING}

A model, representing the cardiopulmonary signal detected using the proposed microwave system, is also presented in this work. The cardiopulmonary signal consists of the combination of two signals representing the heartbeat and the respiration signals. Simulated in Matlab, these two signals are modeled to fit the heartbeat and the respiration signals detected at $5.8 \mathrm{GHz}$ and for a total output power of $-12 \mathrm{dBm}$.

\section{A. Modeling Procedure}

Both heartbeat and respiration signals are modeled with linear equations; i.e. each pulse (beat or breath) is modeled with two concatenated linear functions. The proposed model takes into account three main features: the first feature is to preserve the amplitudes of the measured signals. This guarantees a constant ratio between the amplitude of the heartbeat signal and the amplitude of the respiration signal. The second factor taken into account is the heartbeat rate. This variable is extracted from the original signal and 
considered as a reference to which the heartbeat rate of the filtered signal is compared. The third factor is the heart rate variability. The cardiopulmonary signal is modeled according to the following relation:

$$
\begin{gathered}
Y=\sum_{i=1}^{N} \frac{(-1)^{(i+1)}+1}{2}\left[A_{u}\left(\frac{i+1}{2}\right) X_{u}(i)+B_{u}\left(\frac{i+1}{2}\right)\right]+ \\
\frac{(-1)^{(i)}+1}{2}\left[A_{d}\left(\frac{i}{2}\right) X_{d}(i)+B_{d}\left(\frac{i}{2}\right)\right]
\end{gathered}
$$

where $A u$ and $B u$ represent the coefficients of the up-side curve of a pulse and, $A d$ and $B d$ represent the coefficients of the down-side curve of a pulse. $X_{u}(i)$ and $X_{d}(i)$ represent respectively the durations of the up- and down-sides of a pulse. The sum of these two durations forms the duration of a single pulse. $n(N=2 n)$ is the number of pulses (beats for heart beating and breathes for respiration). For the respiration signal, $A u$ has an average of 0.0234 and a standard deviation of 0.0075 . $B u$ has an average of -8.6255 and a standard deviation of 1.8641. Ad has an average of -0.0259 and a standard deviation of 0.0039 . $B d$ has an average of 9.3009 and a standard deviation of 1.4645 . For the heartbeat signal, $A u$ has an average of 0.0096 with a standard deviation of 0.0015 . Bu has an average of -1.409 and a standard deviation of 0.391 . Ad has an average of -0.0204 and a standard deviation of 0.0028 . Bd has an average of 1.4394 and a standard deviation of 0.3702 . $X u$ has an average of 0.56 seconds and a standard deviation of 0.0624 . $X d$ has an average of 0.28 seconds and a standard deviation of 0.0588 . The values of $X$ are set based on the performed measurements. Hence, the HRV is practically conserved. The measured heartbeat signal and the modeling signal are shown in Fig. 3. Compared to the measured signal, the proposed model shows an accuracy of $100 \%$ in terms of heartbeat rate while it shows a mean relative error of $0.12 \%$ in terms of HRV.

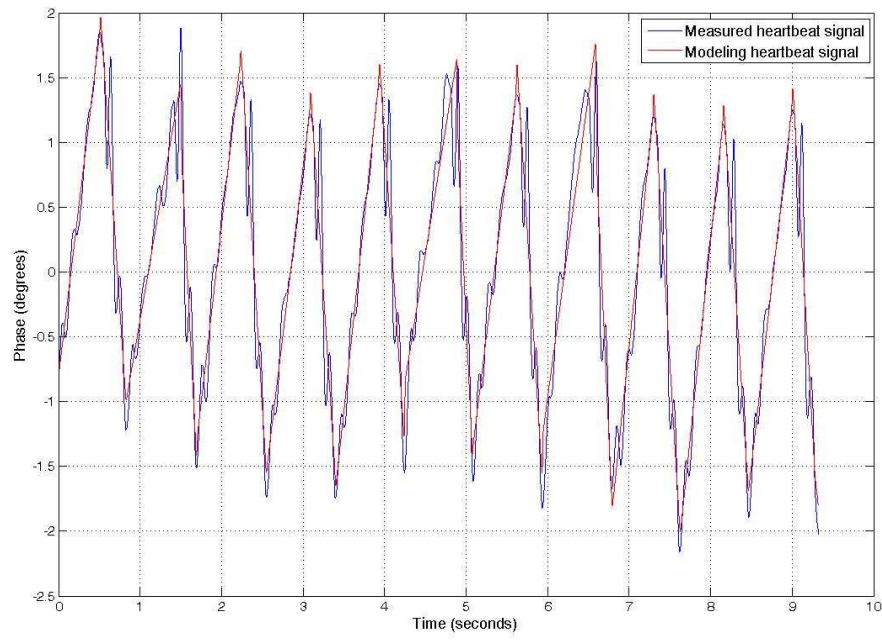

Fig. 3. Phase variations due to heartbeats detected at $5.8 \mathrm{GHz} \mathrm{Vs}$ the modeling heartbeat signal.

\section{B. Processing Procedure}

A white Gaussian noise is added to the combination of the heartbeat signal and the respiration signal. The noise samples are generated as pseudo-random values drawn from a normal distribution with mean zero and standard deviation one $\left(\sigma_{n}=1\right)$. The amplitudes of the heartbeat and the respiration signals are decreased by the same ratio in order to have different SNR levels. Then the separation of the heartbeat and the respiration signals is processed using different techniques at each SNR level. The SNR values are set between 0 and $-20 \mathrm{~dB}$. The relation between the amplitudes of the heartbeat signal and the SNRs is as follows:

$$
\operatorname{SNR}(d B)=20 * \log _{10} \frac{A_{\text {eff }}}{\sigma_{n}}=20 * \log _{10} A_{\text {eff }}
$$

The amplitude of the heart signal is calculated according to $A_{h}=A_{\text {eff }} * \sqrt{3}$. Keeping the same ratio between the amplitude of the respiration signal and the amplitude of the heartbeat signal, the respiration signal is calculated as Ar=Ah/ratio.

For each value of the SNR between 0 and $-20 \mathrm{~dB}$, by $1 \mathrm{~dB}$ step, different processing techniques were used to separate and to compute the heartbeat rate and the HRV. The first method used to determine the mean value of the heartbeat rate is based on the fast Fourier transform (FFT). The second method used to determine the heartbeat rate is based on the detection of the signal's peaks. On the other hand, the heart rate variability requires peak-to-peak detection. Thus, a Peak Detection Method (PDM) is applied to the signals. This technique is based on tracking the maximum value in a sliding window. The size of the window is 2 seconds and the minimum and maximum durations between two consecutive peaks are 0.5 and 1.5 seconds respectively. These values correspond to a heartbeat rate between 30 and 120 beats per minute. Two filtering techniques are used in order to eliminate the respiration signals: the first technique is to apply a high-pass type-1 order-4 Chebyshev filter with 0.6 $\mathrm{Hz}$ cut-off frequency. The second technique is to apply wavelet filter. The wavelet transform with Daubechies wavelets order-4 (db4) is used. The wavelet decomposition of the signal is obtained at level 8 , and then the detail coefficients are reconstructed in order to obtain the heartbeat signal. The FFT is applied to the modeled cardiopulmonary signal, to the Chebyshev filtered signal and to the wavelet filtered signal. However, the PDM is applied to the wavelet filtered signal.

\section{Results: HeARTBEAt RAte AND HEART RATE VARIABILITY}

Wavelet transformation has been applied in different applications in biomedical signal processing $[15,16]$. Due to its capability of providing a multilevel analysis, the wavelet transform shows better performance over classical filters in the separation of the heartbeat signal from the 
cardiopulmonary signals. The use of wavelet transform with $4^{\text {th }}$ order Daubechies wavelets shows the possibility to extract the HRV from the heartbeat signal at very low SNR.

The extraction of the mean of the heartbeat rate upon applying the FFT to the original signal and to the filtered signal shows an average error less than $1 \%$ over all the simulations between 0 and $-20 \mathrm{~dB}$. The extraction of the heartbeat rate upon applying the PDM to the Chebyshev filtered signal shows a mean relative error about $9.1 \%$. However, the extraction of the heartbeat rate upon applying the PDM to the wavelet filtered signal shows $100 \%$ accuracy. On the other hand, wavelet transform shows the possibility to extract the HRV with a mean relative error about $4.5 \%$. Fig. 4 shows the mean relative error in extracting the HRV of the signal after applying the wavelet transform at SNR values between 0 and $-20 \mathrm{~dB}$.

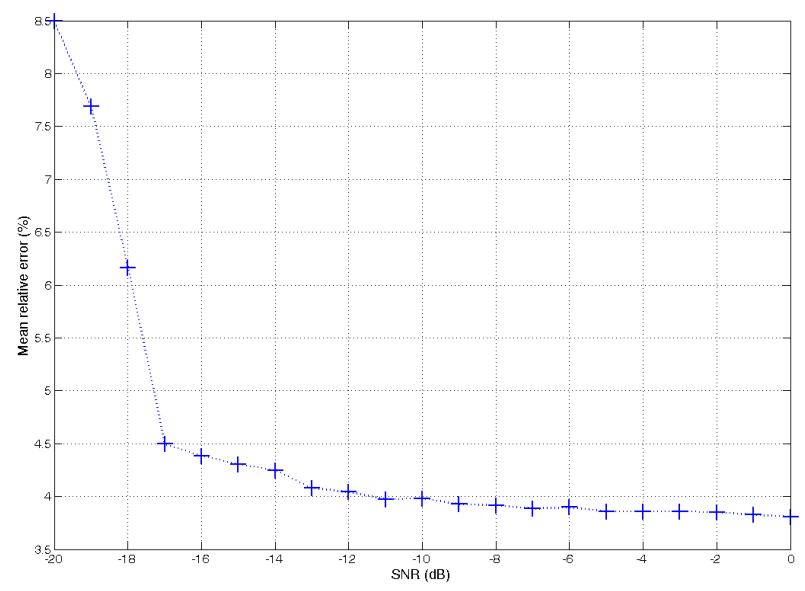

Fig. 4. Mean relative error in extracting the HRV upon applying wavelet transforms to the modeling cardiopulmonary signals.

\section{CONCLUSION AND PROSPECTIVES}

The proposed system shows the possibility to detect the heartbeat activity at different operational frequencies and different power levels. The system was tested at 2.4, 5.8, 10, 16, and $60 \mathrm{GHz}$. Higher sensitivity to small displacements was observed at higher operational frequencies. Operating at $2.4 \mathrm{GHz}$, the proposed system shows the ability to detect the heart activity at radiated power as low as as $-27 \mathrm{dBm}$. The detection of the peaks of the heartbeat signal allows extracting not only the heartbeat rate, but also the heart rate variability.

In order to sustain a practical study that is necessary prior the implementation process, a cardiopulmonary modeling is proposed. This would help in specifying the most accurate processing techniques for both the separation of the cardiopulmonary signals and the extraction of the heartbeat rate with high accuracy. Based on performed measurements, a model representing the cardiopulmonay signals is simulated in Matlab. For different SNR levels, the separation between the respiration signal and the heartbeat signal is acheived. Classic filters allow the detection of the heartbeat rate with high accuracy even at $-20 \mathrm{~dB}$ SNR. However, classic filters lack in providing accurate HRV extraction. On the other hand, wavelet filters show high accuracy (>99\%) in extracting both the heartbeat rate and the heart rate variability.

Our future work will focus on generalizing the proposed model by performing measurements with different subjects and under different breathing circumstances.

\section{REFERENCES}

[1] J. C. Lin, "Microwave sensing of physiological movement and volume change: A review," Bioelectromagnetics (1992), vol. 13, pp. $557-565$.

[2] A. DeGroote, M. Wantier, G. Cheron, M. Estennes, and M. Pavia, "Chest wall motion during tidal breathing," Journal of Applied Physiology (1997), vol. 83, no. 5, pp. 1531-1537.

[3] G. Ramachandran and M. Singh, "Three-dimensional reconstruction of cardiac displacement patterns on the chest wall during the P, QRS, and T-segments of the ECG by laser speckle interferometry," Medical and Biological Engineering and Computing (1989), vol. 27, no. 5, pp. 525-530.

[4] F. Mohammad-Zahed, F. Taghibakhsh and B. Kaminska, "Contactless Heart Monitoring," 2007 IEEE, Session 33: Biomedical Signal Processing-I, pp. 583-585.

[5] K. M. Chen, Y. Huang, J. Zhang, and A. Norman, "Microwave lifedetection systems for searching human subjects under earthquake rubble and behind barrier", IEEE Trans. Biomed. Eng. Jan. 2000, vol. 47, no. 1, pp. 105-114.

[6] A. D. Droitcour, V.M. Lubecke, J. Lin, and O. Boric-Lubecke, "A microwave radio for Doppler radar sensing of vital signs," in IEEE MTT-S Int. Microw. Symp. Dig., May 2001, pp. 175-178.

[7] A. D. Droitcour, O. Boric-Lubecke, V. M. Lubecke, and J. Lin, "0.25 $\mu \mathrm{m}$ CMOS and BiCMOS single chip direct conversion Doppler radars for remote sensing of vital signs," in IEEE Int. Solid-State Circuits Conf. Tech. Dig., Feb. 2002, pp. 348-349.

[8] O. Boric-Lubecke, G. Awater, and V. M. Lubecke, "Wireless LAN PC Card Sensing of Vital Signs," IEEE Topical Conference on Wireless Communication Technology (2003), pp. 206-207.

[9] Y. Xiao, J. Lin, O. Boric-Lubecke, and V. M. Lubecke, "A Ka-band low power Doppler radar system for remote detection of cardiopulmonary motion," presented at the 27th IEEE Annu. Eng. Med. Biol. Soc. Conf., Sep. 1-4, 2005.

[10] Y. Xiao, J. Lin, Boric-Lubecke, and V. M. Lubecke, "Frequency tuning technique for remote detection of heartbeat and respiration using lowpower double-sideband transmission in Ka-band," IEEE Trans. Microw. Theory Tech., vol. 54, no. 5, pp. 2023-2032, May 2006.

[11] V. M. Lubecke, O. B. Lubecke, A. H. Madsen, and A. E. Fathy, "Through-the-wall radar life detection and monitoring," in IEEE International Microwave Symposium, 2007, pp. 769-772.

[12] C. Li and J. Lin, "Random body movement cancellation in doppler radar vital sign detection," IEEE Trans. on Microw. Theory Techn., vol. 56, pp. 3143-3152, 2008.

[13] M. D’Urso, G. Leone, and F. Soldovieri, “A simple strategy for life signs detection via x-band experimental set-up," Progress in Electromagnetics Research C, vol. 9, pp. 119-129, 2009.

[14] D. Obeid, S. Sadek, G. Zaharia, and G. El Zein, "Non-Contact Heartbeat Detection at 2.4, 5.8 and $60 \mathrm{GHz}$ : A Comparative Study," Microwave Opt. Technol. Lett., vol. 51, no. 3, pp. 666-669, March 2009.

[15] M. Akay, "Time frequency and wavelets in biomedical signal processing," IEEE Press, New York, 1998.

[16] S. Mallat, and W.L. Hwang, "Singularity detection and processing with wavelets," IEEE Trans., IT, vol. 38, pp. 617-643, 1992. 